\title{
Integrated image content and metadata search and retrieval across multiple databases
}

\author{
M. Addis ${ }^{1}$, M. Boniface ${ }^{1}$, S. Goodall ${ }^{2}$, P. Grimwood ${ }^{1}$, S. Kim², P. Lewis ${ }^{2}$, K. \\ Martinez $^{2}$, A. Stevenson ${ }^{1}$ \\ ${ }^{1}$ [mja,mjb,pg,as]@it-innovation.soton.ac.uk. IT Innovation, University of Southampton, \\ SO16 7NP, UK. \\ ${ }^{2}$ [sg02r,sk,phl,km]@ecs.soton.ac.uk. Department of Electronics and Computer Science, \\ University of Southampton, SO17 1BJ, UK.
}

\begin{abstract}
This paper presents an updated technical overview of an integrated content and metadata-based image retrieval system used by several major art galleries in Europe including the Louvre in Paris, the Victoria and Albert Museum in London, the Uffizi Gallery in Florence and the National Gallery in London. In our approach, the subjects of a query (e.g. images, textual metadata attributes), the operators used in a query (e.g. SimilarTo, Contains, Equals) and the rules that constrain the query (e.g. SimilarTo can only be applied to Images) are all explicitly defined and published for each gallery collection. In this way, cross-collection queries are dynamically constructed and executed in a way that is automatically constrained to the capabilities of the particular image collections being searched. The application of existing, standards based, technology to integrate metadata and content based queries underpins an open standards approach to extending interoperability across multiple image databases.
\end{abstract}

\section{Introduction}

Museums and galleries often have several digital collections ranging from public access images to specialized scientific images used for conservation purposes. Crosscollection access is recognised as important, for example to compare the treatments and conditions of Europe's paintings, which form a core part of our cultural heritage. The ARTISTE project [1], partly funded by the European Union under the fifth framework, has developed a system for the automatic indexing and cross-collection search and retrieval of high-resolution art images. Four major European galleries are involved in the project: the Uffizi in Florence, the National Gallery and the Victoria and Albert Museum in London, and the Centre de Recherche et de Restauration des Musées de France (C2RMF) which is the Louvre related restoration centre. The ARTISTE system currently holds over 160,000 images and 5,000,000 textual metadata items from four separate collections owned by these partners. 
2 M. AddisP1P, M. BonifaceP1P, S. GoodallP2P, P. GrimwoodP1P, S. KimP2P, P. LewisP2P, K. MartinezP2P, A. StevensonP1P

\section{Overview}

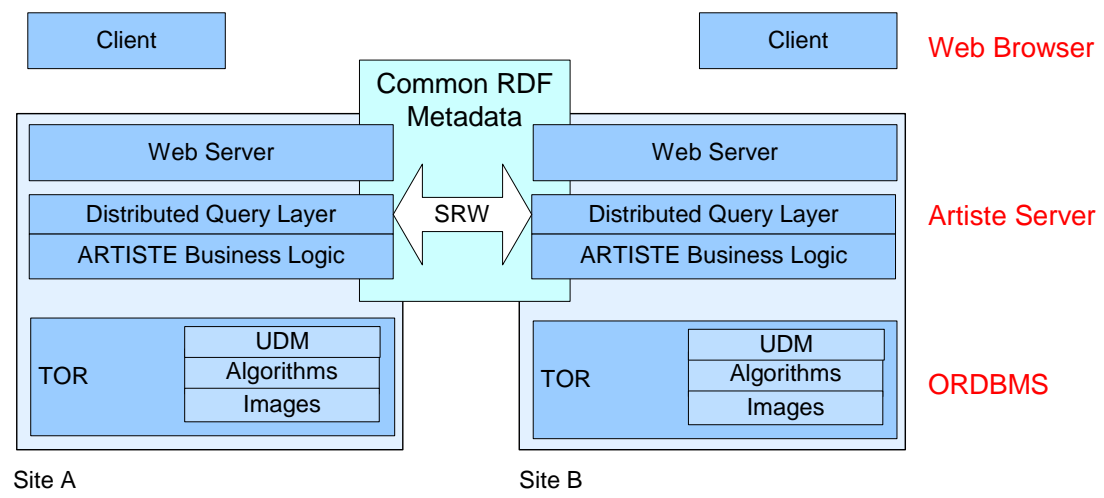

Figure 1 ARTISTE system architecture

Images of the art objects in a museum or gallery collection are held in an Object Relational Database Management System (ORDBMS) from NCR. Images are stored as Binary Large Objects (BLOBS). ARTISTE uses a wide variety of image processing algorithms [2][3][4][5] as the basis of content-based retrieval. Each algorithm is applied to the images in the collection to generate a set of image content descriptors called 'feature vectors'. The feature vectors are then integrated and stored with the text metadata for each image in the database.

When a content-based search needs to be made, the required algorithm is run on the query image to create a query feature vector. For example, the user might have a query image of a particular object that they wish to locate in a collection. Alternatively, the user might have a query image containing a particular range of colours and wish to find images with similar colours in the image collection. The query feature vector is then compared with all the corresponding feature vectors for the images in the collection. The comparison of feature vectors results in a measure of distance between the query image and each image in the collection. The images in the collection are then returned to the user as a series of thumbnails in order of increasing distance.

The use of a multi-tier architecture allows physical distribution of the system components so that cross-collection searching can be performed when the image collections are physically located at each of the gallery sites. This is achieved by having a local image database and ARTISTE server at each site that owns an image collection. The use of open standards such as OAI [6] and SRW [7] provides the basis of interoperability between systems installed at different galleries and allows other software systems, for example digital libraries, to work with ARTISTE. 


\section{Image content based analysis}

One of the aims of the ARTISTE project was not only to apply some standard algorithms for content based image retrieval in a gallery context [19][24], but also, through consultation with the end users, to address requirements for new or modified content based approaches meeting their specific needs. Standard algorithms for colour similarity matching included colour and grey scale histograms and an implementation of the colour coherence histogram [15] which incorporates spatial information. A colour picker applet allows users to retrieve images containing particular colours. These can be selected from a colour patch, or by mixing colours in HSB, RGB, or Lab space. Texture matching was based on energy coefficients in the pyramid wavelet transform [17] using Daubechies wavelets [18]. Other wavelet decompositions and basis functions were implemented and compared along with Gabor filter banks but the incorporated approach was chosen as the best compromise between retrieval accuracy and computational efficiency [20]. Some of the specific requirements for non standard content analysis from the galleries, which were addressed in the project, are summarised below. Our collaborators included groups interested in restoration and some of the requirements were more concerned with, for example, the restoration framework than the art work itself.

- An ability to retrieve images based on a sub-image query i.e. where the query is only a part of the database image. This was addressed by introducing multi-scale variants of some of the standard algorithms. Each database image was divided into 64 by 64 pixel patches, feature vectors were calculated for each patch, the resolution was reduced by a factor of 2 on each axis and the process repeated recursively until the image was represented as nearly as possible by a single patch. Retrieval involved finding the best matching patch across all searched images and gave approximate location information for the matching sub-image. See [5] for further details.

- An ability to handle retrieval using low quality query images such as faxed images of postcards of art works. The problem here was that the query images were essentially binary. Feature vectors for each database image were generated by thresholding a monochrome version at multiple positions across the histogram and using a modified pyramid wavelet transform to represent each resulting binary image. Good retrieval results were achieved [4].

- Analysis and retrieval based on the extent and type of crack patterns (craquelure) in the painting. Progress was made in detecting and classifying different types of crack pattern using ultra violet images to capture the basic crack data, morphological filtering to isolate the cracks and a track following and thinning algorithm to extract the detailed crack patterns. Local and global statistics of the patterns are used to classify the cracks using a fuzzy K-means classifier. The work is documented in [3] and [23].

- Retrieval by shape of the frame of a painting. Some of the galleries had a requirement to retrieve images of paintings by the shape of their border and a border location algorithm was implemented using a snake to close in on the object within an image. The border so obtained was classified using a neural 
4 M. AddisP1P, M. BonifaceP1P, S. GoodallP2P, P. GrimwoodP1P, S. KimP2P, P.

LewisP2P, K. MartinezP2P, A. StevensonP1P

net algorithm to allocate the shape to one of a number of frame shape classes such as rectangular, oval, triptych.

- Characterisation of UV reflectance of paintings (as an indicator of the extent of previous restoration work). Dark regions of a UV image may be indicative of previous restoration work and retrieval and quantification of such areas was a particular goal. It was initially expected that we would be able to automatically threshold the UV image at an appropriate level and extract an appropriate estimate of the proportion of dark spots under UV lighting. However, inconsistencies in UV spot images made automation difficult and a stand-alone tool was developed for the partners to allow them to manipulate the UV images interactively - thereby helping to make their prognosis faster and easier.

Most of the retrieval algorithms used nearest neighbour matching in some multidimensional feature space in order to find good matches to a query. However, since multiple content based retrieval algorithms were to be used in a single search, for example to find the best match in terms of both colour coherence and texture, and also results were to be aggregated when querying across multiple collections, it was necessary to find a way of combining similarity measures. A normalisation procedure was introduced for each class of feature vector which transformed distance measures in that feature space to a probability of finding a better match than the one returned. This was achieved by generating an approximation to the cumulative probability distribution for distances in that feature space by comparing all combinations of a large sample of the images in the collections and fitting the distribution with a polynomial function which could be used to normalise the distances for that feature space. The normalisation was built into the feature matching modules so that when a distance is calculated it is returned as a probability, with zero indicating a perfect match and 1 indicating the worst possible match. Although several assumptions are implicit in this process, it provided a good mechanism for combining content based retrieval processes from the different algorithms and returned a more meaningful estimate of the similarity based on the characteristics of the collections in the system.

\section{Integrated metadata and image content-based analysis}

Current digital library query representations and protocols, such as z39.50 [12], deal entirely with textual metadata. This is not sufficient for multimedia digital libraries such as ARTISTE, where searches can be made on image content as well as textual metadata. In particular, current protocols have the following restrictions.

- There are no methods for specifying image content as a metadata item;

- There are no operators defined relating to image content;

- There are no methods for carrying out searches that result in the execution of image processing algorithms. 
ARTISTE has addressed each of these issues by using RDF and RDFS [13] to define a query ontology [16] which defines and describes the objects, methods and operators required to build a query based on either image content, textual metadata or a combination of the two. All items that can be queried are described by a 'Query Item' hierarchy. A Query Item can be an image, properties of an image (such as colour or shape), or attributes associated with an image (conventional metadata such as textual and numeric items). The operations that can be performed on Query Items are also explicitly defined as Query Operators. Current Query Operators include exact operators (such as equals, less than, etc.) and fuzzy operators (such as similar to). $\mathrm{RDF}$ is also used to define the syntax and semantics for standard metadata terms. Each collection provides a mapping that relates these standard metadata terms to individual database table and column values. Thus queries specified using the RDF contain no concrete mappings to actual database fields. Therefore they can be passed without alteration to multiple gallery sites. At each site it will be translated to SQL and in particular the Dublin Core [8] Title is translated to the appropriate table and column name for the local metadata schema. In this way the ARTISTE system provides the ability to execute queries across multiple, distributed collections without requiring each collection to conform to a standard schema. Benefits of this RDF approach include:

- The use of RDF mapping provides a flexible solution to cross-collection searching;

- Mapping to a common schema allows common semantics to be supported without needing changes to local metadata and schemas;

- Users can be dynamically constrained in their querying through use of a 'Query Context' so that they only request queries that are within the common capabilities of a set of collections;

- Multilingual translation of metadata attribute names allows the user to use their native language when specifying which attributes to search over for multiple collections;

- Free text translation is a possible solution to multilingual searching of metadata content.

\section{Extending Interoperability}

The extensive use of RDF in ARTISTE provides a way to establish common semantics between heterogeneous digital libraries containing image collections. These common semantics include how to perform content-based analysis as well as textual metadata searching. This goes a long way towards interoperability between multiple digital libraries. In fact, this is sufficient to enable cross-collection search and retrieval between ARTISTE systems. However, common semantics are not enough to provide interoperability with third-party systems. To achieve this requires adoption of standards for the process of search and retrieval itself, i.e. use of standard protocols. 
6 M. AddisP1P, M. BonifaceP1P, S. GoodallP2P, P. GrimwoodP1P, S. KimP2P, P.

LewisP2P, K. MartinezP2P, A. StevensonP1P

As stated above, existing information retrieval standards have been traditionally concerned with text based searching. Therefore ARTISTE is participating in an initiative [7] to redesign the primary open standard for interoperability between digital libraries, z39.50, using web technologies such as XML and SOAP. The initiative has proposed a Search and Retrieve Web Service (SRW) based on the z39.50 protocol for searching databases that contain metadata and objects [7]. ARTISTE is one of the early implementers of SRW and has extended the capabilities of SRW to enable image content and metadata based searches over multiple collections. ARTISTE has been working with the z39.50 community to incorporate the ability to deal with content-based searching of images and thus expand international standards of information retrieval.

The Common Query Language (CQL) [25] proposed by the SRW specification supports metadata based querying but makes no provision for content based queries. For example there is no provision to specify image analysers in combination with image operators and the search term is always assumed to be a text string. The limitations of the language were addressed by extending the protocol in those areas where it does not cover image content based querying and by maintaining close contact with the z39.50 community developing the SRW specifications.

The ARTISTE CAQL [14][10] expands CQL to provide support for image content queries by adding image operator (img-op), image analyser (img-analyser) and an image expression (img-exp) to the language.

primary : :=result-set-expression | [index-name rel-op]

adj-expr | index-name img-op img-analyser img-exp

The SRW CQL specification of result-set-expression and index-name remains unchanged in the ARTISTE CAQL. The ARTISTE CAQL further specifies elements necessary to an image content query

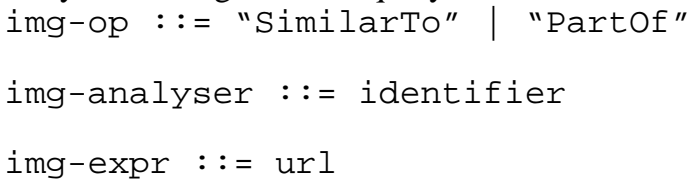

It can be seen from the definition of img-expr above that query images are specified as URLs. The same approach is used for query result images. Some examples of CAQL queries are given below.

dc.Creator contains Vinci and

artisteCore.VisibleLightImage SimilarTo CCV

http://artiste.it -

innovation.soton.ac.uk/test_images/test.jpg

This query combines a Dublin Core 'Creator' metadata search with an image contentbased query that uses the ' $\mathrm{CCV}$ ' algorithm to find images that are 'SimilarTo' the referenced query image 'test.jpg'.

dc.Subject $=$ TEXTILE and artisteCore.VisibleLightImage

Partof MCCV http://artiste.it- 


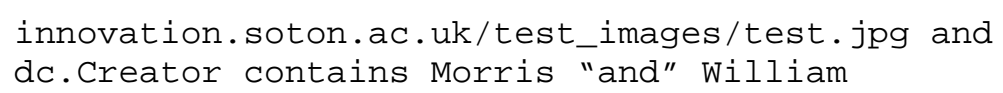

This query combines a textual metadata search involving the Dublin Core attributes 'Subject' and 'Creator' with an image content-based query that uses the 'MCCV' algorithm to find images that have the referenced query image as 'part of' them, i.e. as a sub-image.

\section{Results}

The ARTISTE system provides seamless access to multiple distributed image collections, enabling dynamic searching of high resolution art by image content and metadata [22]. The example query (Figure 2) shows a query combining image content and metadata searching across the Victoria and Albert Museum collection. The query uses the Multi-scalar Colour Coherence algorithm to find larger images containing the specified sub image where those larger images have been marked up as 'Photograph'.

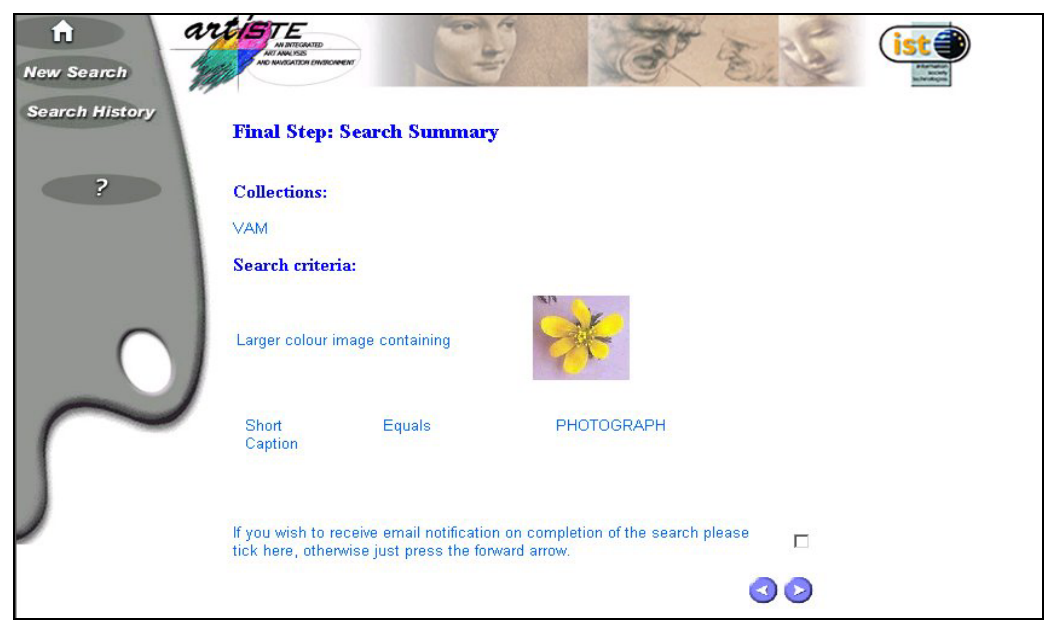

Figure 2 Sample ARTISTE query

The results (Figure 3) show the system finds the photograph containing the query image as well as other photographs containing similar shapes and colours. 
8 M. AddisP1P, M. BonifaceP1P, S. GoodallP2P, P. GrimwoodP1P, S. KimP2P, P. LewisP2P, K. MartinezP2P, A. StevensonP1P

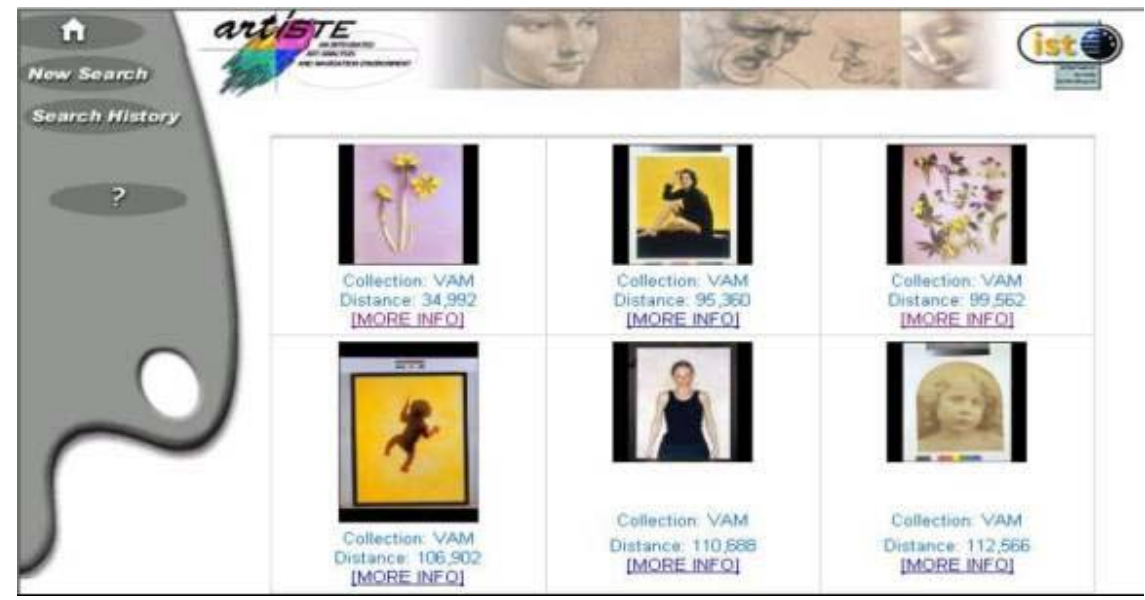

Figure 3 Sample ARTISTE query results

The results of a second query are shown in Figure 4. The query was executed across three collections located variously at the National Gallery, the Uffizi Gallery and C2RMF and the metadata search term 'Dublin Core Creator' was selected from an automatically generated list of only those terms available which can be mapped to fields in each and every one of the selected collections. The results show images returned from each of the collections.

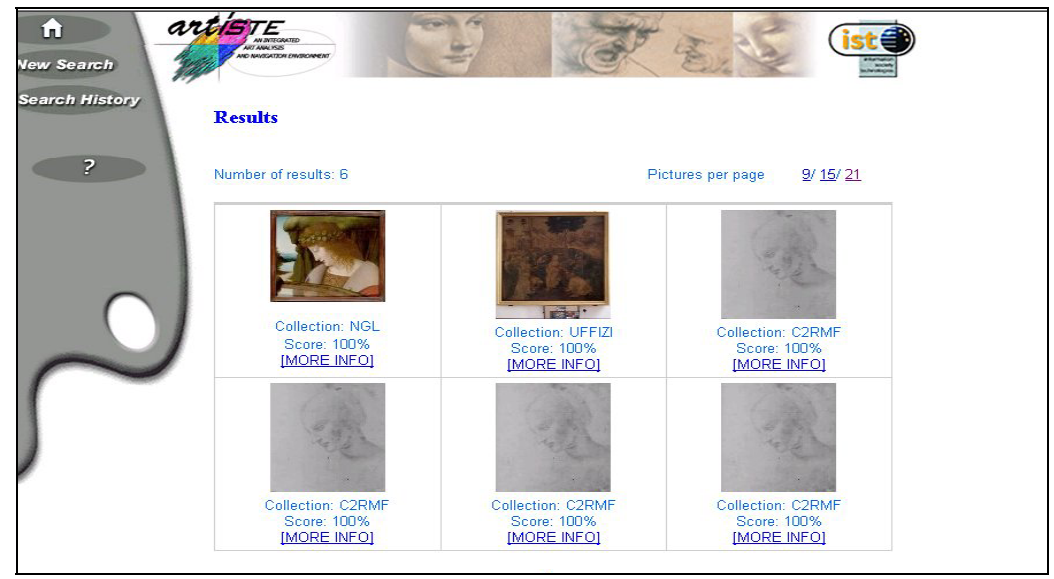

Figure 4 Sample ARTISTE cross collection query results

A demonstration system is available for interested users [9] to test [11] and ARTISTE has also released a standalone client which provides access to the demonstration collection via the SRW interface [26]. 


\section{Observations and future work}

Digital library support for multimedia content-based search and retrieval is in its infancy and is not accommodated by current standards. We believe the full benefit of multimedia search and retrieval can only be realised through seamless integration of content-based analysis techniques. This requires modification to existing standards as well as a review of the use of semantics in achieving digital library interoperability. In particular, machine understandable descriptions of the semantics of textual metadata, multimedia content, and content-based analysis can provide a foundation for a new generation of flexible and dynamic digital library tools and services. In essence, this is the application of Semantic Web [21] techniques to digital library interoperability. ARTISTE has begun this work and it will continue in the SCULPTEUR project [27]. SCULPTEUR aims to extend digital image library technology to support more diverse multimedia objects as well as use evolving semantic web technologies such as OWL to generate, structure and link together metadata in museum collections. Among the challenges the project will face are the storage and retrieval of 3D multimedia objects, the development of algorithms for content-based analysis of 3D objects, and the development of new protocols for interoperability between digital libraries.

\section{Conclusions}

The exploitation of cultural image collections is limited because of a lack of conformance to common schema, and lack of appropriate and convenient access methods. The ARTISTE project has addressed these issues not simply by providing access through a single application but by incorporating into international standards of information retrieval the ability to deal with content based searching. ARTISTE has both used and provided input to standards for metadata and digital image libraries. The significance of the work will be determined by the extent to which it is incorporated into the developing interoperability standards.

\section{Acknowledgements}

The authors are grateful to the European Commission for their support through grants IST-1999-11978 and IST-2001-35372 and to their collaborators on the ARTISTE project (NCR (Dk), Giunti Interactive Labs (I), Uffizi Gallery (I), The National Gallery (UK), C2RMF (FR), and the Victoria \& Albert Museum (UK)) for image data and useful conversations. 
10 M. AddisP1P, M. BonifaceP1P, S. GoodallP2P, P. GrimwoodP1P, S. KimP2P, P. LewisP2P, K. MartinezP2P, A. StevensonP1P

\section{References}

[1]ARTISTE http://www.artisteweb.org

[2]Addis, M., Lewis, P., Martinez, K. "ARTISTE image retrieval system puts European galleries in the picture", Cultivate Interactive http://www.cultivate-int.org/issue7/artiste/

[3]F. S. Abas and K. Martinez (2002) Craquelure Analysis for Content-Based Retrieval. IEEE DSP 2002 conference. July 2002.

[4]M.F.A.Fauzi and P.H.Lewis Query by Fax for Content Based Image Retrieval CIVR, Lecture Notes in Computer Science, vol2383, 91-99,2002 Springer Verlag.

[5]S Chan, K Martinez , P Lewis, C. Lahanier \& J. Stevenson Handling Sub-Image Queries in Content-Based Retrieval of High Resolution Art Images. International Cultural Heritage Informatics Meeting p.157-163. September 2001

[6]Open Archives Initiative http://www.openarchives.org/

[7]ZING Search and Retrieve Web service http://www.loc.gov/z3950/agency/zing/srw/

[8]DublinCore metadata initiative http://www.dublincore.org/

[9]Artiste User Interest Group. Contact: M. Cecil-Wright mcw@it-innovation.soton.ac.uk

[10]"D6.2 Impact on World-Wide Metadata Standards" http://www.itinnovation.soton.ac.uk/artiste/documentation/D6.2.final.pdf

[11]ARTISTE public dissemination system http://artiste.it-innovation.soton.ac.uk/

[12]z39.50 http://lcweb.loc.gov/z3950/agency/

[13]Resource Description Framework http://www.w3.org/RDF/

[14]“D6.1 Distributed Query Layer and Metadata Report” http://www.itinnovation.soton.ac.uk/artiste/documentation/D6.1final.pdf

[15]G Pass, R Zabih, \& J Miller. Comparing Images Using Color Coherence Vectors. MultiMedia, p65-73. ACM, 1996.

[16]Artiste Core schema http://artiste.it-innovation.soton.ac.uk/rdf/ArtisteCore.rdf

[17]S.G.Mallat, A theory for multiresolution signal decomposition: The Wavelet Representation, IEEE Transactions on Pattern Analysis and Machine Intelligence, 11, 674-693, July 1989

[18]I. Daubechies, “The wavelet transform t-frequency localisation and signal analysis”,IEEE Transactions on Information Theory, vol36,pp. 961-1005,1990

[19]J. Cupitt \& K. Martinez, "VIPS: an image processing system for large images”, Proc. SPIE conference on Imaging Science and Technology, San Jose, Vol. 2663, 1996, pp 19-28

[20]M.F.A.Fauzi, “Texture Based Image Retrieval using Multiscale sub-image Matching”, Image and Video Communications and Processing 2003 (to be published).

[21]The Semantic Web http://www.semanticweb.org/

[22]P. Allen, M. Boniface, P. Lewis, K. Martinez "Interoperability between Multimedia Collections for Content \& Metadata-Based Searching”, 11th WWW Conference, Hawaii. 711 May 2002 http://www2002.org/CDROM/alternate/196/index.html

[23]F. Salleh Abas and K. Martinez, "Classification of Painting Cracks for Content-Based Analysis”, Machine Vision Applications in Industrial Inspection XI, 2003 (to be published)

[24]A. W. M. Smeulders, M. Worring, S. Santini, A. Gupta, \& R. Jain. Content-Based Image Retrieval at the End of the Early Years. In Transactions On Pattern Analysis And Machine Intelligence, volume 22 of 12, pages 1349-1380. IEEE, December 2000.

[25]ZING CQL specification http://www.loc.gov/z3950/agency/zing/cql/cql-syntax.html

[26]ARTISTE SRW Service demonstration http://artiste.it-innovation.soton.ac.uk/srw

[27]SCULPTEUR EC IST project number 35372 http://www.sculpteurweb.org 